\title{
Structural sensitivity of superconducting properties of layered systems
}

\author{
A.L. Kuzemsky ${ }^{a, *}$, I.G. Kuzemskaya ${ }^{b}$ \\ a Bogolubov Laboratory of Theoretical Physics, Joint Institute for Nuclear Research, 141980 Dubna, Moscow Region, Russia \\ ${ }^{\mathrm{b}}$ High Pressure Physics Institute, Russian Academy of Sciences, 142092 Troitsk, Moscow Region, Russia \\ Received 4 February 2002; received in revised form 27 February 2002; accepted 12 March 2002
}

\begin{abstract}
Physics of layered systems is studied from the crystal structure point of view. The relationships between structural and superconducting properties are discussed, and particular attention is paid to the layered structure. We discuss the possible role of inequivalent layers and charge transfer interlayer redistribution. By taking into account the modified charge transfer approach a workable model that provides a possibility for comparison of the structural, electronic, chemical factors etc., for the occurrence of superconductivity of layered systems is considered. The present paper analyzes the possible applications of the inequivalent layer model to the mercurocuprate family and provides rationalization to the experimentally observed nonmonotonic "bell"-shaped dependence of critical temperature of a number of layers in the elementary cell.

(c) 2002 Elsevier Science B.V. All rights reserved.
\end{abstract}

PACS: 61.00.00; 61.50.-f; 61.50.Ah; 61.66.-f; 4.20.De; 74.72.Gr

Keywords: Crystal structure; Layered systems; Mercury-based copper oxides; Superconductivity

\section{Introduction}

There is a big variety of layered systems constructed of various compounds, including the cuprate oxides [1,2], with unusual resistive transitions. Many materials, such as high- $T_{\mathrm{c}}$ oxides and artificially stacked superconducting multilayers [3,4], were synthesized and tested. A number of superconducting systems with the layered perovskite

\footnotetext{
* Corresponding author. Fax: +7-096-2165084.

E-mail address: kuzemsky@thsun1.jinr.ru (A.L. Kuzemsky).

$U R L:$ http://thsun1.jinr.ru/ kuzemsky.
}

structure were synthesized. The discovery of superconductivity in $\mathrm{Sr}_{2} \mathrm{RuO}_{4}$ confirmed the conjecture that the presence of copper is not a strict condition for the appearance of superconductivity in the layered perovskite compounds. For example, it was found that $\mathrm{KCa}_{2} \mathrm{Nb}_{3} \mathrm{O}_{10}$ and $\mathrm{KLaNb}_{2} \mathrm{O}_{7}$ had triple and double layered perovskite structures, respectively [5]. Although they are electrically insulating, $\mathrm{Li}$ intercalation drastically reduces resistivity and induces an insulatormetal transition. The $\mathrm{Li}$ intercalated $\mathrm{KCa}_{2} \mathrm{Nb}_{3} \mathrm{O}_{10}$ exhibits a superconducting transition around $1 \mathrm{~K}$. However, $\mathrm{KLaNb}_{2} \mathrm{O}_{7}$ shows no superconductivity down to $0.5 \mathrm{~K}$. Recently, the layered cobalt oxides that are the cobalt analogs of the superconducting 
cuprates were discovered [6]. These compounds provide the possibility for direct comparison with the superconducting cuprates to determine which structural, electronic or chemical factors are required for the occurrence of the high- $T_{\mathrm{c}}$ superconductivity (HTS). While a complete understanding of the role of the layered structure is clearly desirable, a more penetrating insight into this is complicated by many factors that are related to the difficulties of the characterization of the single-phase samples. Additionally, the stacking of layers in a compound as well as the coordination of atoms in the layer, layer thickness, and also symmetry and chemistry play a role in these materials. All these factors, which influence $T_{\mathrm{c}}$, should be taken into account in a proper combination. For instance, the standard charge transfer picture of copper oxides suggests that superconductivity occurs predominantly in the $\mathrm{CuO}_{2}$ planes, while other (intercalated) layers behave as charge reservoirs. However, the arguments were presented [7] that, in principle, it is possible to think that the intercalating metal oxygen layers are not just passive insulating layers, but are "electronically active". However, the subsequent experimental studies on the Y-, Bi- and Tl-based cuprates do not seem to bring a support to such a conjecture in spite of that there exist various conflicting arguments in the literature on this subject. The situation with the $\mathrm{Hg}$-based (mercurocuprate) family of copper oxides is even more complicated because of the big difficulties in the preparation and characterization of the high-quality single-phase samples, and experimental clarification of the properties and the role of the $\mathrm{Hg}-\mathrm{O}$ layers. Mercurocuprates with the perovskite structure represent a very important family of oxides which is extensively studied for their record high-temperature superconducting properties [8-19].

In the present work, we will analyze the different factors that can govern the unique physical properties of the mercurocuprate family $\mathrm{HgBa}_{2} \mathrm{Ca}_{n-1}$ $\mathrm{Cu}_{n} \mathrm{O}_{2 n+2+\delta}$. The dependence of $T_{\mathrm{c}}$ vs. $a$, where $a$ is the in-plane lattice parameter (and, consequently, oxygen content $\delta$ ) has a universal "bell"-shaped character in layered mercurocuprates. The similar bell-shaped character has a dependence of $T_{\mathrm{c}}(n)$ on the number of layers $n$ with the maximum at $T_{\mathrm{c}}$ $(n=3)$. This beneficial effect of the optimal number $n=3$, which induces the maximal $T_{\mathrm{c}}$ in these compounds, is still unclear. In view of these facts, a theoretical approach to determining the critical temperatures for homologous series depending on a number of layers $n$ is desirable. The dependence of a superconducting critical temperature and the formal copper valence on a number of layers $n$ for different members of mercurocuprate family should be analyzed. To do this, in the present study we consider these problems in terms of the workable model of layered superconductors taking into account possible charge redistribution. This leads to rationalization of the observable nonmonotonic bell-shaped dependence of $T_{\mathrm{c}}(n)$ and provides a quantitative explanation of the experiments. It will be shown that the correlations between the copper valency, lattice parameters and extra oxygen contents are the essential factors in the physical behaviour and HTS characterization of the mercurocuprates.

\section{Layered cuprates}

The fabrication of single-phase samples is a complicated problem due to the existence of chemical instabilities in most cuprates, as evidenced by the difficulties in obtaining the samples with the full oxygen stoichiometry. As a result, the synthesis condition should be fine-tuned in the formation of these compounds.

There have been many attempts to classify known HTS cuprates to explain both the formation of specific phases and the occurrence of HTS and correlate $T_{\mathrm{c}}$ with other physical parameters. A number of factors should be taken into account. The model structure of cuprate superconductors shares a common feature in that they are made up of a regular stacking of metal oxide layers and that each repeat unit contains one or more copper oxide layers in which superconductivity takes place. The active components, i.e., $\mathrm{CuO}_{2}$-layers of the compounds with proper doping relate most closely to transport and superconducting properties. It is established that $T_{\mathrm{c}}$ of HTS depends on the carrier concentration $p$ in the $\mathrm{CuO}$-layers. It is also 
known that the carrier distribution may be nonuniform in compounds with several $\mathrm{CuO}_{2}$-layers in the unit cell. The variation of $T_{\mathrm{c}}$ in different HTS families, such as La-, Bi-, Tl- and Hg-based HTS, was studied as a function of doping, pressure and structure and is in general explained by the variation of carrier concentration in the $\mathrm{CuO}_{2}$-layers. It was found that $T_{\mathrm{c}}$ behaves approximately as a parabola with changing hole concentration per copper ion and has a maximum at the optimally doped level [10]. The studies of the mercurocuprate family of high-temperature superconductors were the object of special interest during the last years after their discovery [8-10]. Of particular importance is the question of structural sensitivity or interrelation of crystal structure and superstructure and superconductivity, and the role of anisotropy in layered superconducting cuprates. A comprehensive knowledge of the structure of oxide superconductors is essential in attempting to understand their physical behaviour. According to [11], the concept of the homologous series in the case of cuprate superconductors can be defined as "a family of layered copper oxides that differ from one another only by the number of pairs $(n-1)$ of $\mathrm{CuO}_{2}$ and bare cation planes in the infinite-layer block". One can speak about a homologous series only when the members at least up to $n=3$ have been experimentally found. Each homologous series discovered till now has at least one member whose $T_{\mathrm{c}}$ exceeds $100 \mathrm{~K}$. The mercurocuprate family $\mathrm{HgBa}_{2} \mathrm{Ca}_{n-1} \mathrm{Cu}_{n} \mathrm{O}_{2 n+2+\delta}$ (the symbolic notation is $\mathrm{Hg}-12(n-1) n)$ is of special interest because it culminates the fascinating features of HTS and are still the highest $T_{\mathrm{c}}$ representatives of cuprates [12]. The actual issue for mercurocuprate family is to understand the exact role of layered structure and most important parameters that govern the highest value of transition temperature of these materials in spite of that the mechanism for actual superconductivity remains less clear.

The concept of a homologous series [11,13-16], which in the case of mercurocuprates plays a especially important role, raises a natural question about the dependence of a superconducting critical temperature of this family of layered copper oxides on the number of pairs $(n-1)$ of $\mathrm{CuO}_{2}$ and bare cation planes in the infinite-layer block [17]. In

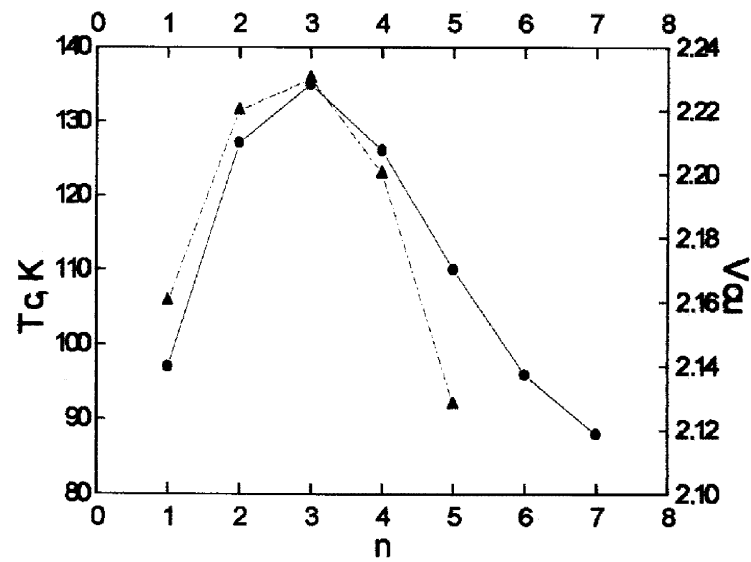

Fig. 1. Dependence of the superconducting critical temperature and copper valence on number of layers $n$ according to data from Table 2. Full line is the critical temperature and dashed line is valence.

other words, the main interest in this respect is dependence $T_{\mathrm{c}}(n)$ [16-19] (Fig. 1).

On the other hand, there seems to be a close relationship between the average copper valence and the phase produced in the high-pressure synthesis of mercurocuprates $[9,10]$. This is related with the oxidation of the $\mathrm{CuO}_{2}$-layers. According to [9], the formal copper valence for different members of the mercurocuprate family is equal to $v_{\mathrm{Cu}}=2(n+\delta) / n$. The values of $\delta$ obtained from neutron scattering experiments lead to the conclusion that extra oxygen content and, consequently, the copper valence and lattice parameter depend on the number of $\mathrm{CuO}_{2}$-layers (Fig. 1) and on heat treatment of the samples [10].

The $T_{\mathrm{c}}(n=1,2)$ can be changed by reducing of oxidation treatments, contrary to $T_{\mathrm{c}}(n=3)$ which is not so strongly influenced by high pressure oxygen treatment. This may reflect the important fact that in the Hg-1223 structure, the distribution of charges between the two type of $\mathrm{CuO}_{2}$-layers is different. Such a structural specific feature, which includes the interplay of two "active" elements of different kind, could be responsible for this behaviour. In the simplest case it could be the planes and the chains, but as regards the inequivalent $\mathrm{CuO}_{2}$-layers in multilayer structure, the inner and outer $\mathrm{CuO}_{2}$-layers can have different charge carrier density. Thus, the correlation between the copper 
valence, lattice parameters and extra oxygen contents becomes then important.

\section{Crystal structure of cuprates and mercurocup- rates}

All cuprate HTS have a layered structure. They are oxides and can be considered as a stack of layers of cation-oxygen and cations [10]. The generic feature is the presence of $m(\mathrm{AO})$-layers inserted on the top of $n \mathrm{CuO}_{2}$-layers. In other words, layers of these compounds can be grouped into two blocks: the charge-reservoir block and active block. The charge-reservoir block provides sources of charge carriers for the active block which is considered the main component for superconductivity in the compound.

The homologous series with the common formula $\mathrm{A}_{m} \mathrm{M}_{2} \mathrm{R}_{n-1} \mathrm{Cu}_{n} \mathrm{O}_{x}$, where $\mathrm{A}=\mathrm{Bi}, \mathrm{Tl}, \mathrm{Pb}, \mathrm{Cu}$, $\mathrm{Hg}, \ldots, \mathrm{M}=\mathrm{Ba}, \mathrm{Sr}, \mathrm{R}=\mathrm{Ca}$ or RE-cation, $m=1$, 2 and $n=1,2,3, \ldots$ can be fabricated. The resulting structure is the sequence (or "stack") of the $\left(\mathrm{CuO}_{2}\right),(\mathrm{AO}),(\mathrm{MO})$ and $(\mathrm{R} \aleph)$ layers (here $\aleph$ is the anion vacancy). There are different compounds with $1 \leqslant \mathrm{CuO}_{2} \leqslant n$. If $n>1$, then these layers are intercalated by $(n-1)$ layers $(\mathrm{R} \aleph)$. The crystal structure of the compounds $\mathrm{A}_{m} \mathrm{M}_{2} \mathrm{R}_{n-1} \mathrm{Cu}_{n} \mathrm{O}_{x}$ can be represented schematically as

$$
-\left[(\mathrm{MO})(\mathrm{AO})_{m}(\mathrm{MO})\right]\left[\left(\mathrm{CuO}_{2}\right)\left\{(\mathrm{R} \aleph)\left(\mathrm{CuO}_{2}\right)\right\}_{n-1}\right]
$$

Such a sequence of layers in the structure of a material should fulfill the geometric and charge stability criteria that are related with the valence, ionic radii, crystal coordination, etc. Here (MO) are the dielectric layers and $\mathrm{CuO}_{2}$ are the conducting layers in the "NaCl"-type block and perovskite block, respectively. The known geometric criterion for the stability of the perovskite structure reads

$t=\frac{\left(R_{\mathrm{M}}+R_{\mathrm{O}}\right)}{\sqrt{2}\left(R_{\mathrm{Cu}}+R_{\mathrm{O}}\right)}$

Here $t$ is the tolerancy factor and for the ideal situation this factor is $t=1$. The $\mathrm{CuO}_{2}$-layers and the (MO)-layers fit each other ideally if

$$
\frac{d(\mathrm{MO})}{d(\mathrm{Cu}-\mathrm{O})} \sim \sqrt{2}
$$

In the various $\mathrm{HTS}$ cuprates the $(\mathrm{Cu}-\mathrm{O})$-length is $1.90 \leqslant d(\mathrm{Cu}-\mathrm{O}) \leqslant 1.98 \AA$. So, the ideal $(\mathrm{M}-\mathrm{O})$ length should be $2.69 \leqslant d(\mathrm{M}-\mathrm{O}) \leqslant 2.80 \AA$ A. Approximately, the same distance range is necessary for the (A-O)-length in the (AO)-layer. So, both the layers (AO) and (MO) that constitute the " $\mathrm{NaCl}$ "-type block are responsible for the stability of the structure and provide necessary hole concentration for the conduction band. The geometric criteria mean that the structure is stable under the condition when the cation-oxygen distance in each given plane is commensurate with the same distance in the neighboring planes. Some of geometric data [18] are listed in Table 1. From Table 1 it is clear that the most suitable M-cations are the barium, strontium and RE-cations. For those elements in Table 1 that may serve as the A-cations, the ionic radii are much lesser as compared to the corresponding ones for strontium or barium and their using will lead to the strong incommensurability. Sometimes, in these layers, an additional amount of oxygen can be embedded. As a result, an ideal coordination of the A-cations by the oxygen atoms can be reached.

Various techniques for the the synthesis and fabrication of high-quality HTS materials are available. Recently, there has been great progress in the synthesis of high-quality mercurocuprate samples [22-38]. Homologous series [11] $\mathrm{HgBa}_{2}$ $\mathrm{Ca}_{n-1} \mathrm{Cu}_{n} \mathrm{O}_{2 n+2+\delta}$ have been fabricated using the high-pressure high-temperature synthesis that seems to be the efficient and workable method to

Table 1

Some geometric data

\begin{tabular}{lll}
\hline Item $\backslash$ data & Size of A-cation $\AA$ & Size of M-cation $\AA$ \\
\hline $\mathrm{Hg}^{2+}$ & 1.10 & \\
$\mathrm{Ce}^{3+}$ & 1.03 & \\
$\mathrm{Bi}^{3+}$ & 0.99 & \\
$\mathrm{Tl}^{3+}$ & 0.88 & \\
$\mathrm{Ti}^{4+}$ & 0.61 & \\
$\mathrm{~V}^{5+}$ & 0.54 & \\
$\mathrm{Cr}^{3+}$ & 0.62 & \\
$\mathrm{Mo}^{6+}$ & 0.67 & \\
$\mathrm{~W}^{6+}$ & 0.54 & \\
$\mathrm{Re}^{6+}$ & 0.52 & 1.25 \\
$\mathrm{Sr}^{2+}$ & & 1.47 \\
$\mathrm{Ba}^{2+}$ & & \\
\hline
\end{tabular}


Table 2

Critical temperatures, oxygen contents and parameters of elementary cell for mercurocuprate family (literature data)

\begin{tabular}{llllllll}
\hline Hg-12(n-1)n & Hg-1201 & Hg-1212 & Hg-1223 & Hg-1234 & Hg-1245 & Hg-1256 & Hg-1267 \\
\hline$n$ & 1 & 2 & 3 & 4 & 5 & 6 & 7 \\
$T_{\mathrm{c}}, \mathrm{K}$ & $97-98$ & 127 & 135 & 127 & 110 & 107 & 88 \\
$\delta^{\text {opt }}$ & 0.08 & 0.22 & $0.27(4)$ & $0.4(1)$ & $0.32(2)$ & - & - \\
$\delta$ (interval) & $0.008-0.19$ & $0.08-0.35$ & $0.19-0.44$ & $0.4-0.6$ & $0.32(2)$ & - & - \\
$a, \AA$ & 3.879 & 3.855 & 3.852 & 3.854 & 3.852 & 3.8533 & $3.847-851$ \\
$c, \AA$ & 9.509 & 12.652 & 15.82 & 19.01 & 22.10 & 25.5 & 28.7 \\
Apex $(\mathrm{Cu}-\mathrm{O}), \AA$ & - & - & $2.741(6)$ & $2.82(2)$ & $2.85(1)$ & - & - \\
Cu-valence & - & - & - & $+2.20(5)$ & - & - & - \\
\hline
\end{tabular}

produce high-quality $\mathrm{Hg}$-superconducting samples [22-38]. The synthesis of $n=1,2, \ldots, 8$ of the $\mathrm{Hg}$ based homologous series was performed by this and other techniques $[39,40]$ (some data and parameters are listed in Table 2). The dependence of $T_{\mathrm{c}}$ on the lattice distance $a$ for $n=1-5$ members of the family is shown in Fig. 2. The dependence of $T_{\mathrm{c}}$ vs. the $a$-parameter for these phases exhibit a bellshaped behaviour. Hence, this behaviour seems to reflect an intrinsic property of the mercurocuprate crystal lattice structure. We analyzed the interrelation of in-plane lattice distance, number of layers and $T_{\mathrm{c}}$ (Figs. 3-5). When one plots the lattice distance $a$ against the mercurocuprate family members (Fig. 5) it appears that this dependence manifests the minimal value $a_{0}$. Therefore, these pictures show that the key factor in search for the best material parameters is to find the optimal values among all possible ones. Progress in sample

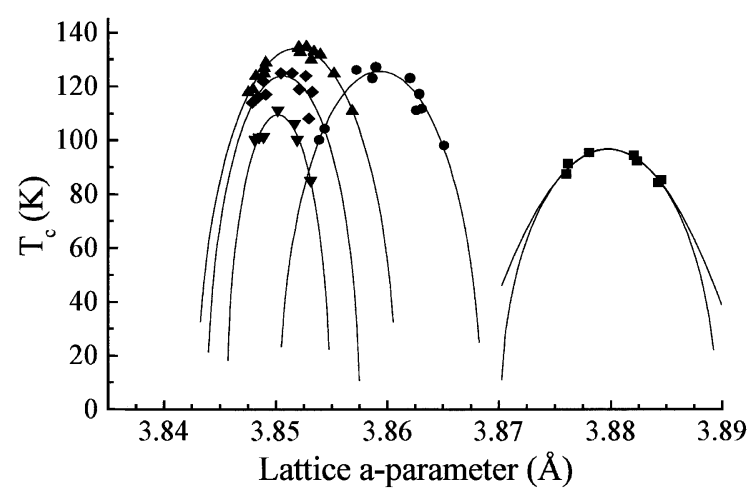

Fig. 2. Dependence of the superconducting critical temperature

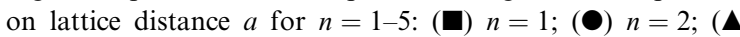
$n=3$; $(\diamond) n=4 ;(\nabla) n=5$; (cf. [32]).

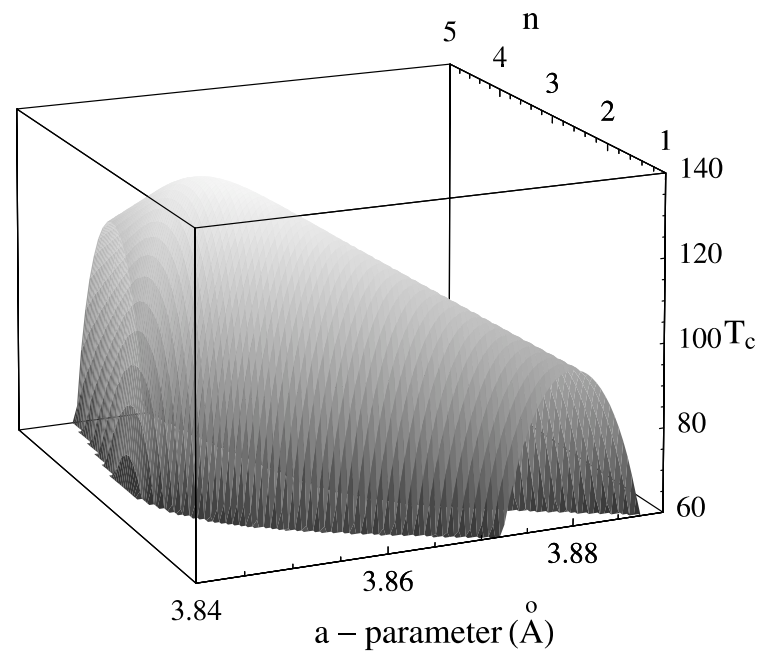

Fig. 3. Dependence of $T_{\mathrm{c}}$ vs. $n$ and $a$.

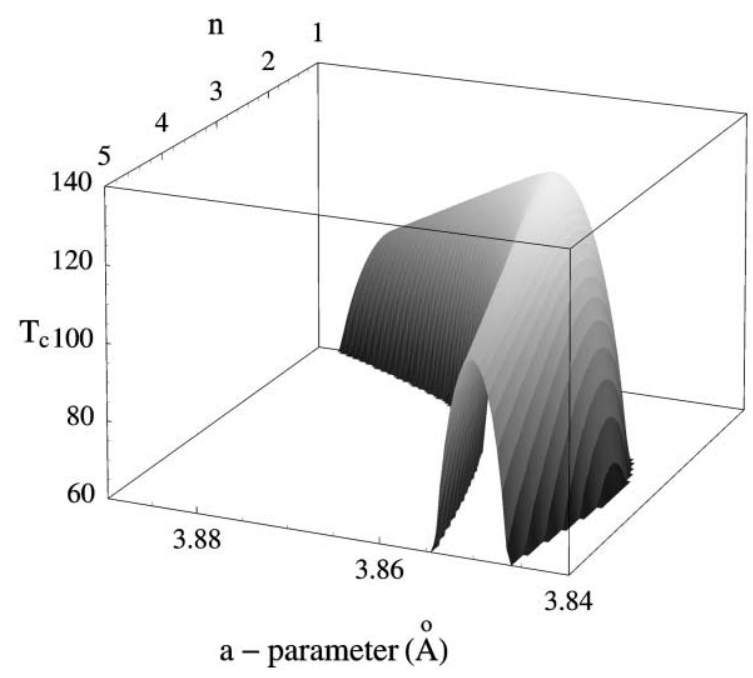

Fig. 4. Dependence of $T_{\mathrm{c}}$ vs. $n$ and $a$. 


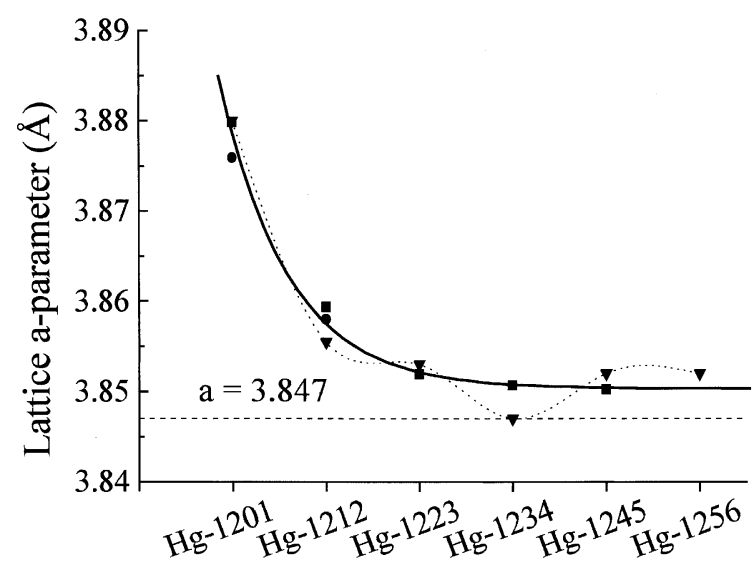

Fig. 5. Dependence of lattice parameter $a$ vs. number of $\mathrm{CuO}_{2}$ layers and the minimum value of in-plane lattice parameter $a_{0}$ :

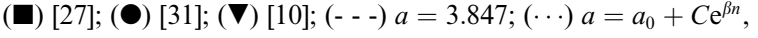
$a_{0}=3.85032, C=0.11113, \beta=0.73044$.

fabrication has recently allowed one to detect signatures of the superconducting behaviour in the mercurocuprates in a more clear way and leads to unambiguous evidence for the specific role of the layered structure in these materials [10]. The highest superconducting transition temperature at ambient pressure was observed for the third $(n=3)$ of the $\mathrm{Hg}$-based copper-mixed oxide series $\mathrm{HgBa}_{2} \mathrm{Ca}_{2} \mathrm{Cu}_{3} \mathrm{O}_{8+\delta}(\mathrm{Hg}-1223)$ with $T_{\mathrm{c}}$ (onset) at $135 \mathrm{~K}$ (see Fig. 1). This feature of the highest $T_{\mathrm{c}}$ for $n=3$ is analogous to that occurring in the Tl- and Bi-based series [20,21].

Detailed structural studies of the mercurocuprates have been performed recently [41-45]. Mercurocuprates were found to possess a layered structure similar to that of other cuprate HTS with a stacking sequence of layers

$$
\begin{aligned}
& -\left[(\mathrm{BaO})\left(\mathrm{HgO}_{\delta}\right)(\mathrm{BaO})\left(\mathrm{CuO}_{2}\right)(n-1)\right. \\
& \left.\quad \times\left\{(\mathrm{Ca} \aleph)\left(\mathrm{CuO}_{2}\right)\right\}\right](\mathrm{BaO})-
\end{aligned}
$$

with $n \mathrm{CuO}_{2}$-layers per unit cell. The structure of the family of mercurocuprates can be viewed [10] as consisting of the $\mathrm{Ca}_{n-1} \mathrm{Cu}_{n} \mathrm{O}_{2 n}$ block and the $\mathrm{Hg}-\mathrm{O}_{\delta}$ block which plays a role of a reservoir of charge. The width of the "NaCl" block is fixed and equal to $5.5 \AA$. The width of the perovskite block is $d=4.0+3.2(n-1) \AA$. The parameter $c$ is equal to $c=9.5+3.2(n-1) \AA$.
Unfortunately, mercurocuprates have been produced, as a rule, not in their optimum doping state. This requires some additional treatment to achieve their highest temperature. There is an important difference between the mercurocuprates and the thallium analogues [20]. One of the main differences is that connected with the partially occupied oxygen sites in the region between the $\mathrm{CuO}_{2}$ planes, occupancy for which in mercurocuprates is very small. Thus, the doping state of mercurocuprates can be controlled by changing the excess oxygen content. It is also important to note that for the mercurocuprate family the unilayer, bilayer, trilayer etc. dependence of physical properties shows a different behaviour as regards anisotropy (two- or three-dimensional nature) [26, 29]. The normal state electronic properties are determined by the state of itinerant charge carriers and it is of particular importance to investigate transport characteristics of the mercurocuprate oxides as well [30-32].

\subsection{The properties of the $\mathrm{Hg}-\mathrm{O}$ plane}

According to [9], an important and general structural feature of the mercurocuprates is that the apical $\mathrm{Cu}-\mathrm{O}$ distances are larger than the corresponding distances in other cuprates $(\sim 2.80$ $\AA$ ). Contrary to other cuprates where the $\mathrm{CuO}_{2}$ layers are slightly buckled, the $\mathrm{Cu}$ and $\mathrm{O}$ atoms forming the $\mathrm{CuO}_{2}$-layers in the mercurocuprates are nearly coplanar. These structural features could be responsible for the highest transition temperature as well. The existence of the $\mathrm{Hg}-\mathrm{O}$ layer is the most essential structural feature of the mercurocuprates. The properties of the $\left(\mathrm{HgO}_{\delta}\right)$ layer still are not finally clear and need additional studies. One has also to take into account that for the $\mathrm{Hg}^{2+}$ cations the dumbbell coordination by the anions (with the interatomic distance $\sim 2 \AA$ ) is more preferable. The bonding $\mathrm{Hg}-\mathrm{O}$ in the plane of the layer $\left(\mathrm{HgO}_{\delta}\right)$ is very weak and the oxygen atoms in this layer are disposed at the distance $\sim 2.7 \AA$. As a result, the $\mathrm{Hg}-\mathrm{O}$ bonds do not influence essentially the commensurability of the (MO)- and (AO)-layers. The only incommensurability in the crystal structure of $\mathrm{HgBa}_{2} \mathrm{Ca}_{n-1}$ $\mathrm{Cu}_{n} \mathrm{O}_{2 n+2+\delta}$, that still persists, is one between the 
$\mathrm{CuO}_{2}$ - and (MO)-layers. This provides minimal distortions of the crystal structure of the mercurybased copper oxides. As regards the crystalchemistry specificity of the $\mathrm{Hg}^{2+}$ cations in comparison with $\mathrm{Bi}^{3+}$ and $\mathrm{Tl}^{3+}$, the dumbbells-like coordination is more preferable than the octaedric coordination. There are big discrepancies in the estimation of the population factor of the oxygen $\left(\mathrm{O}_{\delta}\right)$, and the position parameters and the population factor of the mercury atoms. Moreover there is a possibility of substituting of the mercury atoms by the copper atoms or even the carbon atoms.

The problem of $\mathrm{Cu}$-substitution for $\mathrm{Hg}$ in the mercury-based cuprates is an important and not fully understood question of their crystal chemistry. Such a substitution was first conjectured in [33] in the structural studies on powder neutron diffraction on $\mathrm{Hg}-1201$. They interpreted the large Debye-Waller factor of the $\mathrm{Hg}$ site as an indication of partial replacement of $\mathrm{Hg}$ by $\mathrm{Cu}$ and refined about $7(2) \%$ of substitution. This can lead to the placing of two extra oxygen atoms due to the square coordination of the $\mathrm{Cu}$ cations [10]. These extra oxygen atoms provide additional hole doping for the $\mathrm{CuO}_{2}$-layers. Other groups gave further evidence for this substitution for the same system. They reported the sample with $16 \% \mathrm{Cu}$-substituted. In [34], it was observed that for the compound $\mathrm{Hg}-1223$ the $\mathrm{Hg}$-positions were partially substituted by the $\mathrm{Cu}$ atoms with the fraction $16.1 \% \mathrm{Cu}$-substituted replacement and $\delta=0.190$. In [35], the corresponding fraction of the copper atoms was measured as $13 \%$ in $\mathrm{Hg}-1212$ and $18 \%$ in $\mathrm{Hg}-1223$. Further evidence was brought about in [36] (see, however [37]). There are some arguments to think that the replaced $\mathrm{Cu}$ is in the $(+1)$ oxidation state, but this is still a conjecture. Such a layer $\left(\mathrm{HgO}_{\delta}\right)$ with the partially substituted $\mathrm{Hg}$-atoms by the copper atoms can become, in principle, weakly conducting. It can be also the superconducting with a very low critical temperature. To summarize, the real physical structure and properties of the $\left(\mathrm{HgO}_{\delta}\right)$ layer is the very important factor for the HTS characterization of the mercury-based cuprates; additional studies of its role and significance are highly desirable.

\section{Role of layered structure in cuprates}

\subsection{Layered superconductor models}

The problem of theoretical calculation of the superconducting critical temperature of layered superconductors in the context of copper oxides has previously been investigated to explain the variation of $T_{\mathrm{c}}$ with the number $n$ of adjacent $\mathrm{CuO}_{2}$ planes present in the various superconducting copper oxide compounds [46]. The approach based on a two-dimensional model for the electronic structure of $\mathrm{CuO}_{2}$ sheet was proposed in $[47,48]$. It was related with the statement that a $2 \mathrm{D}$ electronic band always shows a logarithmic singularity in the density of states. For $n$ sheets, the density of states in two dimensions was proposed to be $N=n N_{0}$ and the effective electron-phonon interaction $\lambda=n \lambda_{0}$. The law $T_{\mathrm{c}}(n)=T_{0} \exp \left[-1 / \sqrt{n \lambda_{0}}\right]$ was derived. Even for such an oversimplified model this formula was valid only for small $n$ 's. Another mean-field approach was proposed in [49], where it was insisted on that the average spacing between the $\mathrm{CuO}_{2}$-layers and not merely the number of layers per unit cell determined the critical temperature. A more detailed theory was proposed in [50] which gives the monotonic increase in $T_{\mathrm{c}}(n)$ and upper limit for mercurocuprate family $T_{\mathrm{c}}(n=\infty)=$ $153 \mathrm{~K}$. The interlayer effects in $\mathrm{Hg}$-based cuprates under high pressure for samples with $n=1-6$ sublayers in the unit cell was considered in [51]. The theory of the interlayer tunneling of holon pairs was used and applied to $n>4$ cases, and agreement and disagreement with experimental results was analyzed. Jover et al. [52] considered the mercurocuprates up to $n=4$ on the basis of the model approach of charge redistribution of holes among the various $\mathrm{CuO}_{2}$-layers. They found out that for $n \geqslant 3$ this distribution is highly inhomogeneous. This can lead to observable nonmonotonic $n$ dependence of $T_{\mathrm{c}}(n)$. To summarize, an additional extensive studies on the layer dependence of $T_{\mathrm{c}}(n)$ in a wide hole-doping range are desirable.

\subsection{Phenomenological models}

The phenomenological approach to layered copper oxides was developed by many authors. 
Inequivalent layers in the phenomenology of cuprates were considered in [53] in terms of the proximity effect between the superconducting and insulating layers forming the unit cell of various copper oxides. The model treated the observed positive curvature in the temperature dependence of $H_{\mathrm{c} 2}^{\perp}$ and did not tackle the calculation of transition temperature. Subsequently, different authors used several distinct approaches to realize this idea of inequivalent layers. It is interesting to note that the notion of distinct "active" elements in the structure (type of plane, or chain) in layered cuprates was formulated in the early stage of the studies of these materials even with some exotic model statements that adjacent $\mathrm{Cu}-\mathrm{O}$-layers have negative Josephson coupling, forcing the order parameter to change sign from one layer to another [54]. The variation of the transition temperature with the number of copper oxide layers per unit cell was studied in [55], in the various series of layered HTSs. A phenomenological model incorporating interactions of adjacent and alternate CuO-layers was used. The model was then extended to include the interactions of all layers within a unit cell. However, while the dependence of $T_{\mathrm{c}}$ vs. $n$ was significantly modified, the observed nonmonotonicity of the transition temperature was not explained.

A special variant of the Ginsburg-Landau (GL) model for intrinsic layered superconductors was developed in [56]. In this continuous model the spatial dependent GL coefficients $a$ and $b$ have been assumed to describe the inhomogeneity caused by the layered structure. The spatial dependence of the order parameter reflect the layered structure consisting of superconducting and normal (or weakly superconducting) layers. Thus, this model describes the situation in the cuprates in which the order parameter takes a maximum value at $\mathrm{CuO}_{2}$ layers and takes small at the other ones. Using this model the upper critical field in the directions parallel and perpendicular to the layers has been calculated.

The workable phenomenological approach to calculation of transition temperature of layered cuprates has been proposed in [57]. The physical idea was that there are distinct "active" elements in the multilayer structure. Each active element, denoted by $l$ in the structure, is associated with a GL order-parameter field $\psi_{l}(r)$. Each of these $\psi_{l}(r)$ has some distinct "bare" transition temperature $T_{l}^{0}$ in that structure. The full GL theory is a generalization to coupling order parameters of the seminal work of Lawrence and Doniach (LD) $[58,59]$. The GL functional of the LD model is

$$
\begin{aligned}
F=\sum_{l} & \int \mathrm{d}^{2} x\left(\frac{\hbar^{2}}{2 m}\left|\left(\nabla_{l}-\mathrm{i} \frac{2 e}{\hbar c} \vec{A}_{l}\right) \psi_{l}\right|^{2}\right. \\
& \left.+\frac{\hbar^{2}}{2 M s^{2}}\left|\psi_{l}-\psi_{l+1}\right|^{2}+a_{l}\left|\psi_{l}\right|^{2}+\frac{1}{2} b_{l}\left|\psi_{l}\right|^{4}\right)
\end{aligned}
$$

where $m$ and $M$ are the effective masses in the $a-b$ plane and along the $c$-axis, respectively, $a_{l}=a_{l}^{0}\left(T / T_{l}^{0}-1\right)$. The simplified version of the GL theory considers the competing order-parameter fields as spatially homogeneous and the temperature region just near the critical temperature. In the normal state, $T>T_{\mathrm{c}}$ all order parameters are zero. For $T<T_{\mathrm{c}}$ some order parameters $\psi_{l} \neq 0$ for minimum free energy. Near $T_{\mathrm{c}}$, if the coherence length perpendicular to the layers extends over many layers, than the system acts as a bulk anisotropic superconductor.

In the LD model the layered structure is explicitly described by two-dimensional superconducting sheets stacked at a regular interval with the nonsuperconducting spaces between the sheets so that the Josephson coupling is effective. The LD theory is valid for disturbances in which $\psi_{l}$ varies slowly on the scale of $s$ and close to $T_{\mathrm{c}}$. The approach of paper [57] has demonstrated that for polytype multilayer copper oxide systems it is possible to obtain the increasing $T_{\mathrm{c}}(n)$, where $n$ is a polytype number $n=2,3, \ldots, \infty$, with upper limits for $T_{\mathrm{c}}$. In this paper, we apply this line of reasoning (with suitable modifications) for the mercurocuprate family.

\section{Generalized Lawrence-Doniach model}

As stated above, the study of the superconducting properties of layered structures is based on the LD approach [58]. According to this approach, 
we assume that the free-energy density per unit cell in the superconducting state, relative to that in the normal state in the zero field, can be written in the form

$$
\begin{aligned}
f(\vec{r})= & \sum_{i}^{n}\left[\alpha_{i}(T)\left|\Psi_{i}(\vec{r})\right|^{2}+\beta\left|\Psi_{i}(\vec{r})\right|^{4}\right. \\
& \left.+\frac{1}{2 m}\left|\left(-\mathrm{i} \hbar \nabla+\frac{2 e \vec{A}}{c}\right) \Psi_{i}(\vec{r})\right|^{2}\right] \\
& +\sum_{\langle i j\rangle} \eta_{i j}\left|\Psi_{i}(\vec{r})-\Psi_{j}(\vec{r})\right|^{2}
\end{aligned}
$$

where $\Psi_{i}(\vec{r})$ is the GL order parameter of the $i$ th layer, $\nabla$ acts in the $x-y$ plane, $\vec{A}$ is the appropriate vector potential, $\alpha$ and $\beta$ are the usual GL parameters, $\eta_{i j}$ is the positive Josephson interlayer coupling and $\langle i j\rangle$ means the neighbor pair layers summation. The coefficient $\alpha_{i}(T)$ for the $i$ th layer has the usual form

$\alpha_{i}(T)=\alpha_{i}^{\prime} \frac{\left(T-T_{i}^{0}\right)}{T_{i}^{0}}$

where $T_{i}^{0}$ is the critical temperature of the $i$ th layer.

Here, we confine ourselves to the case $\Psi_{i}(\vec{r})=$ $\Psi_{i}(r)$ and $\vec{A}=0$. Near $T_{\mathrm{c}}$, the contribution of $\beta\left|\Psi_{i}\right|^{4}$ is negligible. With this simplification the free-energy density can now be written as

$f=\sum_{i}^{n} \alpha_{i}(T)\left|\Psi_{i}\right|^{2}+\sum_{\langle i j\rangle} \eta_{i j}\left|\Psi_{i}-\Psi_{j}\right|^{2}$

This equation is effective in the quasi-isotropic form, as we have transformed all mass parameters into one parameter $\alpha$. The GL (mean-field) equations may be obtained by minimizing (or, more correctly, extremizing [57]) $f$ with respect to variations in $\Psi_{i}$, yielding

$$
\begin{aligned}
\frac{\delta f}{\delta \Psi_{i}^{*}}= & \left(\alpha_{i}+\eta_{i-1 i}+\eta_{i-1}\right) \Psi_{i} \\
& -\left(\eta_{i-1 i} \Psi_{i-1}+\eta_{i+1} \Psi_{i+1}\right)=0
\end{aligned}
$$

The resulting secular equation is of the form

$$
\left|\left(\alpha_{i}(T)+\eta_{i-1 i}+\eta_{i i+1}\right) \delta_{i j}-\eta_{i j} \delta_{i j \pm 1}\right|=0
$$

According to [57,58], the transition temperature is the largest root. Eq. (6) can be rewritten in the form

$$
\begin{aligned}
& \left|\left(T-T_{i}^{0}+\frac{\eta_{i-1 i}}{\alpha_{i}^{\prime}} T_{i}^{0}+\frac{\eta_{i i+1}}{\alpha_{i}^{\prime}} T_{i}^{0}\right) \delta_{i j}-\frac{\eta_{i j}}{\alpha_{i}^{\prime}} T_{i}^{0} \delta_{i j \pm 1}\right| \\
& \quad=0
\end{aligned}
$$

or as

$$
\begin{aligned}
\operatorname{det}(T I-M) & =0 \\
\text { where } M_{i j}= & \left(T_{i}^{0}-\frac{\eta_{i-1 i}}{\alpha_{i}^{\prime}} T_{i}^{0}-\frac{\eta_{i i+1}}{\alpha_{i}^{\prime}} T_{i}^{0}\right) \delta_{i j} \\
& +\frac{\eta_{i j}}{\alpha_{i}^{\prime}} T_{i}^{0} \delta_{i j \pm 1}
\end{aligned}
$$

The problem then is to find the maximal eigenvalue of the matrix $M$.

\subsection{Identical layers}

Let us consider the simplest case, the system of $n$ identical layers $\left(T_{i}^{0}=T^{0}, \alpha_{i}^{\prime}=\alpha^{\prime}, \eta_{i j}=\eta\right)$. For cuprates this approximation means that the $\mathrm{CuO}_{2}$ planes are equivalent and the only active factors in determining the critical temperature. This case was considered in [57] where the diagonal tunnel terms $\eta\left|\Psi_{i}\right|^{2}$ were omitted. The corresponding form of the secular equation is

$\operatorname{det}(T I-M)=0, \quad M_{i j}=T^{0} \delta_{i j}+\frac{\eta}{\alpha^{\prime}} T^{0} \delta_{i j \pm 1}$

Further, we observe that the explicit form of the equation for determining the eigenvalues of $M$ is

$\Delta_{1}(T, n)=\left|\begin{array}{cccc}\alpha & -\eta & & \\ -\eta & \alpha & -\eta & \\ & \ldots & \ldots & \ldots \\ & -\eta & \alpha & -\eta \\ & & -\eta & \alpha\end{array}\right|=0$

For this monolayer stack of planes the analytical solution is of the form

$T=T^{0}+\frac{2 \eta T^{0}}{\alpha^{\prime}} \cos \left(\frac{k}{n+1} \pi\right), \quad k=1, \ldots, n$

The numerical representation of Eq. (10) is shown in Fig. 6. Hence, $T_{\mathrm{c}}$ is an increasing monotonic function of $n$

$$
T_{\mathrm{c}}=T^{0}+\frac{2 \eta T^{0}}{a^{\prime}} \cos \left(\frac{\pi}{n+1}\right)
$$




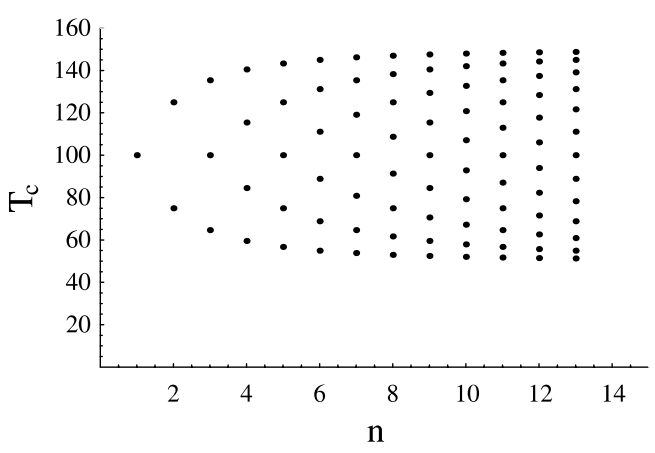

Fig. 6. Roots of BL equation (det $|M|=0): T_{0}=100, a^{\prime}=1$, $\eta=0.25$.

A similar expression

$T_{\mathrm{c}}=T^{0}+T^{\prime} \cos \left(\frac{\pi}{n+1}\right)$

has been derived in [50]. However, in [60] the slightly different model has been studied where the diagonal terms were retained. With this modification Eq. (10) takes the form

$$
\begin{aligned}
\Delta_{2}(T, n) & =\left|\begin{array}{cccc}
\alpha+\eta & -\eta & & \\
-\eta & \alpha+2 \eta & -\eta & \\
& \ldots & \ldots & \ldots \\
& -\eta & \alpha+2 \eta & -\eta \\
& & -\eta & \alpha+\eta
\end{array}\right| \\
& =0
\end{aligned}
$$

yielding the analytical solution

$T=T^{0}+\frac{2 \eta T^{0}}{\alpha^{\prime}}\left[\cos \left(\frac{k-1}{n} \pi\right)-1\right], \quad k=1, \ldots, n$

In Fig. 7 the numerical representation of this equation has been plotted. We therefore observe that within the approach of [60] the critical temperature (maximal solution) of all $n$-layer systems does not depend on $n$ and is equal to the critical temperature of a single layer $T_{\mathrm{c}}=T^{0}$. According to [57], omitting the diagonal terms in the free-energy density leads to a renormalization of the critical temperature. We have

$$
\Delta_{2}(T, n)=\left(T-T^{0}\right) \Delta^{\prime}(T, n-1)
$$

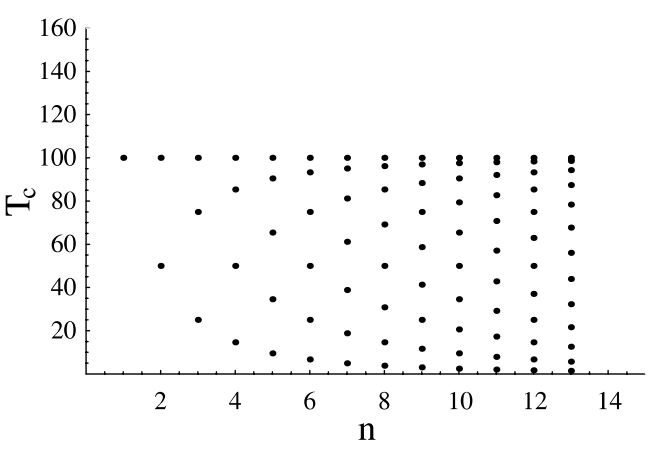

Fig. 7. Roots of MNY equation (det $|M|=0): T_{0}=100, a^{\prime}=1$, $\eta=0.25$.

$$
\Delta^{\prime}(T, n)=\left|\begin{array}{cccc}
\alpha+2 \eta & -\eta & & \\
-\eta & \alpha+2 \eta & -\eta & \\
& \ldots & \ldots & \ldots \\
& -\eta & \alpha+2 \eta & -\eta \\
& & -\eta & \alpha+2 \eta
\end{array}\right|
$$

We make the transformation

$$
\left\{\begin{array}{l}
T \rightarrow T+\frac{2 \eta T^{0}}{\alpha^{\prime}} \\
\Delta_{1}(T, n) \rightarrow \Delta^{\prime}(T, n)
\end{array}\right.
$$

As a result we obtain

$\Delta_{2}(T, n)=\left(T-T^{0}\right) \Delta_{1}\left(T-\frac{2 \eta T^{0}}{\alpha^{\prime}}, n-1\right)$

We observe that an additional solution $T=T^{0}$ exists now, which is the largest one, i.e., $T_{\mathrm{c}}=T^{0}$. The transition temperature for an $n$-layered sample, according to [57], is determined as the maximum eigenvalue of the $n$-dimensional Jacobi (tridiagonal) matrix. For a system composed of $n$ identical layers, where all parameters are identical, this theory leads to expression [57] for $T_{\mathrm{c}}$ providing that the largest solution is $T=T^{0}$ and, as a consequence, $T_{\mathrm{c}}(n)$ is an monotonic increasing function of $n$ with the upper limit $T_{\mathrm{c}}(n=\infty)=141 \mathrm{~K}$.

Nevertheless, the model properties related to these effects do not give the observable bell-shaped form of $T_{\mathrm{c}}(n)$. It is therefore of considerable interest to take into account the interlayer effects of charge redistribution to fit the experimental data. 


\section{Interlayer effects in layered superconductors}

\subsection{The charge redistribution}

The charge carriers in active $\mathrm{CuO}_{2}$ planes are the fundamental degrees of freedom which are primarily responsible for essential physics [62]. The problem of how doping modifies the charge and spin distribution of the system is not well understood for HTS. It was mentioned in literature that the distribution of charge among the $\mathrm{CuO}_{2}$-layers should be understood [62] in order to describe adequately the variation of $T_{\mathrm{c}}$ with the number of $\mathrm{CuO}_{2}$-layers per unit cell in layered cuprates with three or more $\mathrm{CuO}_{2}$ planes.

The carrier concentration $p$ is a crucial factor determining the critical temperature $T_{\mathrm{c}}(p)$ [52]. It was supposed that in HTS a charge redistribution may occur between the reservoir block and active block under various conditions like applied high pressure or with changing the composition, such as oxygen stoichiometry. In the multilayer structures there is a possibility of charge redistributing between the layers within a unit cell, thus leading to models of "inequivalent" $\mathrm{CuO}_{2}$-layers. The inequivalent layer models reveal essential physics arising from the sensitivity to the transition of one of the subsystems to the superconducting state so that $p_{i}$ should rearrange when the system becomes superconducting. In the considered here case of the mercurocuprate family with $n \geqslant 3$, it is the holes in inner and outer $\mathrm{CuO}_{2}$ planes for which charge redistribution transfer with the "reservoir" block seemed to be different.

\subsection{Role of inequivalent $\mathrm{CuO}_{2}$-layers}

As was mentioned above, experimentally it was shown that the highest critical temperatures were reached in structures with a larger number of charge carrier density $p$, i.e., the number of holes per $\mathrm{CuO}_{2}$ layer and copper atom. The empirical equation relating these quantities has the following form:

$T_{\mathrm{c}}(p)=T_{\mathrm{c} \max }\left(1-\beta\left(p-p_{\max }\right)^{2}\right)$

with $\beta \approx 40$ and $p_{\max } \approx 0.2$. However, the superconducting transition temperature depends not only upon the hole density, but also on the number of $\mathrm{CuO}_{2}$-layers (Fig. 1). As a first approximation it is reasonable to suppose that a number of holes is constant and a distribution of these holes between active layers is homogeneous. Then, the interplay of both factors, the plane number $n$ and density of holes $p$ determine $T_{\mathrm{c}}(p, n)$. There is an optimal value for the number of $\mathrm{CuO}_{2}$-layers which maximizes $T_{\mathrm{c}}$. In other words, within this picture the $\mathrm{CuO}_{2}$-layers would be effectively overdoped for smaller values of $n$ and would be underdoped for larger values [52-61]. As was shown in [61], using a simple electrostatic model the charge distribution in multilayer structures with $n \geqslant 2$ may not be homogeneous. In this case, the active $\mathrm{CuO}_{2}$-layers are essentially inequivalent. In spite of this fact, the interplay of both factors, the plane number $n$ and density of holes $p$, determines the observed behaviour of $T_{\mathrm{c}}(p, n)$, leading to an optimal value of $n$ at which a maximum is reached.

Determination of the distribution of holes among the different $\mathrm{CuO}_{2}$ layers of the multilayer cuprate structure has attracted attention of both experimenters and theorists. Various model theoretical estimations were proposed [52-61]. The main task is to determine a fraction $x$ of the total number of holes $\delta$ transferred from the chargereservoir layers (in the case of mercurocuprates of the $\mathrm{Hg}-\mathrm{O}$ layers) which resides on the inner $\mathrm{CuO}_{2}$ layers and the fraction $(1-x)$ of this which resides on the outer $\mathrm{CuO}_{2}$-layers. Here $0 \leqslant x \leqslant 1$. We thus see that the charge carrier density of the inner and outer $\mathrm{CuO}_{2}$-layers is equal to $x \delta /(n-2)$ and $(1-x) \delta / 2$ for inner and outer $\mathrm{CuO}_{2}$-layers, respectively. The electrostatic energy balance requires that $p^{\text {inner }}+2 p^{\text {outer }}=\delta$ for $n=3$. Within the inequivalent layer picture each of the $\mathrm{CuO}_{2}$-layers has its intrinsic $T_{\mathrm{c}}$ value. It was further speculated that the outer $\mathrm{CuO}_{2}$-layers become superconducting for $\delta$ values remarkably lower than the inner ones. This means that the inner $\mathrm{CuO}_{2}$-layers cannot be doped properly in order to become superconducting. This model of the metallic conducting layers [61] has been modified in [62] where the point charge model has been considered. Contrary to the metallic model, the point charge model is based on the ionic picture and the electrostatic energy is replaced with the Madelung energy. 
According to this approach, for small values of $\delta$ most of the charge carriers are retained on the inner $\mathrm{CuO}_{2}$-layers. The charge is transferred to the outer $\mathrm{CuO}_{2}$-layers for a large enough value of $\delta$. As a result, the behaviour of charge transfer can be quite different in these scenarios.

\section{3. $T_{c}$ and the charge carrier density}

In general, the problem of calculating the carrier density dependence of the critical temperature is rather nontrivial. For the BCS superconductor $T_{\mathrm{c}}$, as a function of the charge carrier density $p$ can be roughly approximated by the form [60]:

$T_{\mathrm{c}}(p)=T^{0} \mathrm{e}^{-g / p}$

where $T^{0}$ and $g$ are the fitting parameters. In the structural model [60] this relation has been modified as

$T_{\mathrm{c}}(p)= \begin{cases}T^{0} \frac{p}{p_{0}}, & 0<p<p_{0} \\ T^{0}, & p_{0}<p\end{cases}$

where $T^{0}$ and $p_{0}$ are the relevant fitting coefficients.

Let us consider the stack of active $n$-layers. First, we shall assume that the distribution of hole density $p$ among these layers is homogeneous, i.e., $p_{i}=p / n$. We shall then examine the critical temperature of a single layer $T^{0}$. It is given by the BCS-type Eq. (21) or (22) $T_{i}^{0}=T_{\mathrm{c}}\left(p_{i}\right)$.

\subsection{Inequivalent layer models}

As we have discussed above, the idea of the inequivalent $\mathrm{CuO}_{2}$-layers in cuprates has recently attracted a lot of attention as a factor influencing the transport and superconducting properties of these substances. There is no final and generally accepted picture of the charge transfer in cuprates and its interlayer redistribution. The complexity of their chemical composition and crystal structure dictates that this charge redistribution is also very complex. According to the results of [61], which are based on thallium family, the interlayer charge redistribution is highly inhomogeneous, while other studies [52,62] give the arguments for a nearly homogeneous distribution. On the other hand, the band structure calculations [63], numerical simulation [64] and crystal-chemistry analysis $[15,16]$ suggest the inhomogeneous distribution, but opposite to paper [61].

Since the situation is unclear, we shall analyze various possibilities. We note that the inequivalent layer approach can be realized in a few different ways. Let us first consider the Birman-Lu (BL) [57] approach:

1. It is possible to consider the different parameters $T^{0}$ and $\alpha^{\prime}$ for inner and outer layers.

2. It is possible to consider the charge redistribution by introducing $x$ which is the ratio of the total charge of the inner layers to the total charge of the outer layers.

3. It is possible to introduce the fraction $x$ as a ratio of the total charge of a single inner layer to the total charge of a single outer layer.

Our numerical calculations $T_{\mathrm{c}}(n)$ for all three cases show that the charge redistribution leads to the observable nonmonotonic behaviour. The suitable refinement, which should be made, will be considered in the next section.

\subsection{The $n-m$ layered model}

In the structural model we adopt in the present paper [60], (MNY model) a periodic layered system, whose unit cell contains two kinds ( $a$ and $b$ ) of layers ("active" elements) is considered. In what follows, the layered structure of mercurocuprates is modeled by stack of $n$-layers of type $a\left(\mathrm{CuO}_{2}\right.$ layers) and $m$ layers of type $b$ in an elementary cell. The first and $n$th layers of type $a$ have the same order parameter $(1 \leqslant a \leqslant n$ and $1 \leqslant b \leqslant m)$. Thus, we can incorporate in this formulation the presence of two types of layers (active elements). It is possible in this model (as well as in the continuous model [56]) that the amplitude of the order parameter at weakly superconducting layers is small while superconductivity at strongly superconducting layers is well developed.

Thus, for the periodic system we have an infinite-layer lattice with the unit cell consisting of the two types of layers, $a$ and $b$. The parameters of the $a$-layer are denoted by $\left(\alpha_{a}^{\prime}, T_{a}\right)$, and of the $b$-layer by $\left(\alpha_{b}^{\prime}, T_{b}\right)$. For simplicity, we take that $\eta_{i j} \equiv \eta$. Due to the symmetry of the problem the number 
of parameters is reduced. The order parameter is periodic in a lattice and the order parameters of the 1 st and $n$th layers are equal to $\Psi_{a 1}$, etc. As a result, the number of independent order parameters of the $a$-type will be $v=[n+1 / 2]$ where [z] is the largest integer that does not exceed $z$. The number of independent order parameters for the $m$ layers is given by $\mu=[m+1 / 2]$. As a result, the total number of the independent parameters of ( $n-$ $m$ ) layered model is $v+\mu$. The secular equation, which properly determines a critical temperature as a maximum solution $T_{n}^{\max }$ of this periodic $n-m$ layered system, has the following form:
The numerical solution of these equations with reasonable parameters show that $T_{\mathrm{c}}$ of the whole system is raised with the number $n$ and reduced with the number $m$ (cf. Ref. [60]). This behaviour reflects the fact that the amplitudes of the order parameters for the $a$-layers are larger than those for the $b$-layers. (It is necessary to note that the present model could be refined to reduce the transition temperature of the $b$-type layers practically to zero.) This interplay of two order parameters of two kinds of active elements is one of the most important properties of the present consideration.

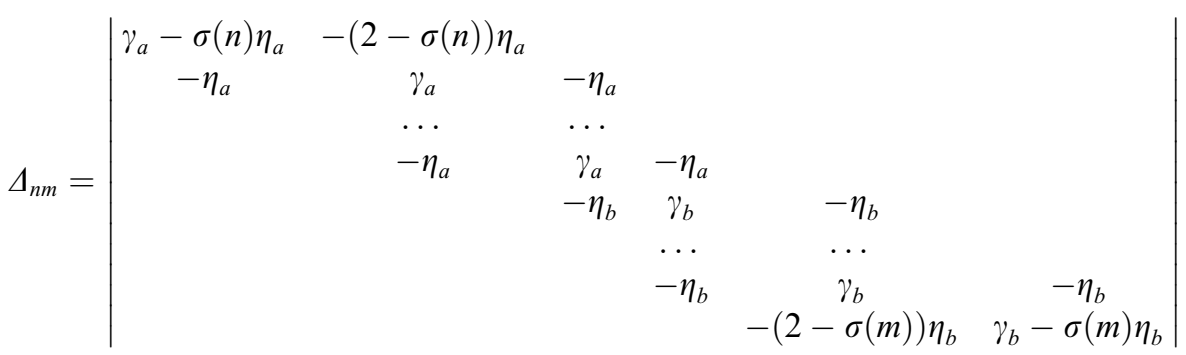

where $\sigma(k)=\left\{\begin{aligned} 1 \quad k & =\text { even, } \\ \gamma_{a} & =T-T_{a}+2 \eta_{a}, \quad \eta_{a}=\frac{\eta}{\alpha_{a}^{\prime}} \\ 0 \quad k & =\text { odd }, \\ \gamma_{b} & =T-T_{b}+2 \eta_{b}, \quad \eta_{b}=\frac{\eta}{\alpha_{b}^{\prime}}\end{aligned}\right.$

$\Delta_{n m}$ is the determinant of a real tridiagonal matrix $(v+\mu) \times(v+\mu)$. The maximum solution will be denoted by $T_{n}^{m}$. We suppose that the parameters $\alpha_{a}^{\prime}$, $T_{a}, \alpha_{b}^{\prime}, T_{b}$ do not depend on the crystal structure, i.e., on $n$ and $m$. Taking this fact into account we find out that all solutions of the secular Eq. (23) will be real and possess the following properties [60]:

1. $T_{b} \leqslant T_{n}^{m} \leqslant T_{a}$

2. $T_{n}^{m}<T_{n+1}^{m}$

3. $T_{n}^{m}>T_{n}^{m+1}$

4. $\lim T_{n}^{m}=T_{a}$

5. ${\stackrel{n}{T_{b}}<}_{m}<\lim _{m \rightarrow \infty} T_{n}^{m}<T_{a}$

\subsection{Effect of structure on the density of carriers}

To get the nonmonotonic bell-shaped dependence of $T_{\mathrm{c}}(n)$, we can construct a simple "toy" model for the structural effects on the charge carrier density. Suppose $n$-layers of type $a$ all have the uniform distribution of charge among them. To fit the data, we take a semiempirical dependence of $T^{0}$ on charge $p_{i}$ in the a-layer in the form of (22). When comparing both the BL and MNY models it is necessary to take into account that in the BL model $\alpha=\alpha^{\prime}\left(\left(T-T_{\mathrm{c}}\right) / T_{\mathrm{c}}\right)$, while in the MNY model $\alpha_{a}=\alpha_{a}^{\prime}\left(T-T_{\mathrm{c}}\right)$.

To describe the experimental data more fully, we attempted to improve the MNY model by taking into the consideration the dependence of $T_{\mathrm{c}}$ not only on $n$, but also on the oxygen contents $\delta$. This was mainly motivated by the fact that maximal values of $T_{\mathrm{c}}$ are different for different members of the mercurocuprate family. 
The following modifications of the MNY model have been made. We suppose that the $b$-type layers with weak superconductive properties possess the following constant parameters $\alpha_{b} i^{\prime}=\alpha_{b}^{\prime}$. The value of $T_{b} i=T_{b}(i=1, \ldots, m)$ is nearly zero. The interlayer tunneling parameter $\eta$ was taken as a constant (in principle, it is possible to take into account different values of $\eta_{a}, \eta_{b}$ и $\eta_{a b}$ ). As we have mentioned above, the dependence of $T_{\mathrm{c}}(\delta)$ has a universal form (cf. [44])

$T_{\mathrm{c}}=T_{\mathrm{c}}^{\max }\left(1-82.6\left(p-p_{0}\right)^{2}\right)$

where $T_{\mathrm{c}}^{\max }$ is the maximal value of $T_{\mathrm{c}}$ at the optimal doping $p=p_{0} \sim 0.16$. It is reasonable to suppose that the charge, which was transferred from the $b$-layers to $a$-layers, is related to the oxygen content $\delta$ by $p=2 \delta$; the charge is distributed among the $a$-layers uniformly: $p_{k}=p / n, \quad(k=$ $1, \ldots, n)$. To proceed further, we adopt the following dependence of a single-layer $T_{\mathrm{c}}$ on the density of charge carriers in this layer $p_{k}$

$T_{\mathrm{c}}\left(p_{k}\right)= \begin{cases}T_{0} \mathrm{e}^{-g / p_{k}}, & 0<p_{k}<p_{0} \\ T_{0} \mathrm{e}^{-g /\left(2 p_{0}-p_{k}\right)}, & p_{0}<p_{k}<2 p_{0} \\ 0, & 2 p_{0}<p_{k}\end{cases}$

where $T_{0}$ and $p_{0}$ are the fitting parameters. The parameter $\alpha^{\prime}$ is the same for all $a$-layers: $\alpha_{a_{k}}^{\prime}=\alpha_{a}^{\prime}$. The results of numerical calculations are plotted in Fig. 8 .

\subsection{Nonuniform redistribution of charge among a-layers}

To rationalize the observable nonmonotonic bell-shaped dependence $T_{\mathrm{c}}(n)$, it is therefore necessary to take into account the interlayer effects of charge redistribution. At the moment, we do not have an effective microscopic mechanism which should explain the nature and provide a certain amount of charge interlayer redistribution. We approximate it by the following simplified form of charge redistribution:

$$
\begin{aligned}
& p_{k}=\frac{2 \delta(1-x)}{n_{\text {out }}} \\
& p_{l}=\frac{2 \delta x}{n-2}
\end{aligned}
$$

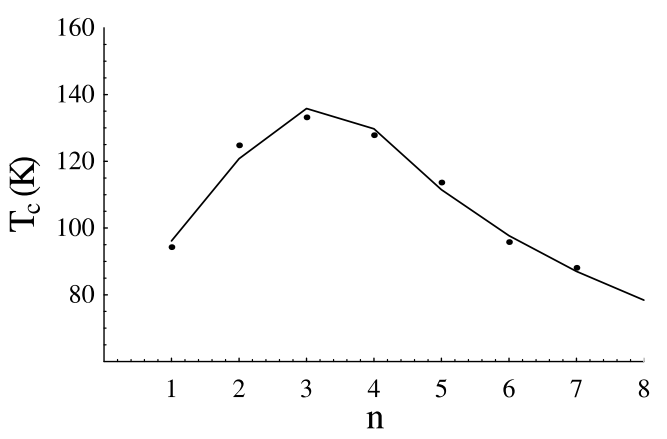

Fig. 8. $T_{\mathrm{c}}$ vs. number of $\mathrm{CuO}_{2}$-layers, MNY model: MNY type $T_{\mathrm{c}}$ vs. charge density dependence: $T_{0}=197.3, \alpha_{a}^{\prime}=0.0045$, $T_{b}=48.5, \quad \alpha_{b}^{\prime}=0.0032, \quad \eta=50, \quad p=1, \quad p_{0}=0.286, \quad m=3$, $\chi^{2}=40.3673$.

where $p_{k}$ is the density of carriers in the outer $a$ layers, $k=1, n ; p_{l}$ is the density of carriers in the inner $a$-layers, $l=2,3, \ldots, n-2, n-1 ; n_{\text {out }}$ is the number of outer layers ( 1 or 2$) ; x$ determines the degree of charge redistribution. In this approximation, the charge in the inner layers is distributed uniformly. As a result, the observable nonmonotonic bell-shaped dependence $T_{\mathrm{c}}(n)$ has been reproduced.

We have plotted in Figs. 9 and 10 the numerical results for $T_{\mathrm{c}}$ on $\delta$ within the model considered here for $n=1,2,3,4,5$ and various parameters $\left(T_{b}=0, \alpha_{a}^{\prime}=\alpha_{b}^{\prime}=0.22, p_{0}=0.214, m=3\right.$ ); (the experimental data are also shown as $(\triangle) \mathrm{Hg}-1201$; $(\diamond) \mathrm{Hg}-1212 ;(\nabla) \mathrm{Hg}-1223 ;(\square) \mathrm{Hg}-1234 ;(\times) \mathrm{Hg}-$ $1245[10])$. The observable model effect consist in

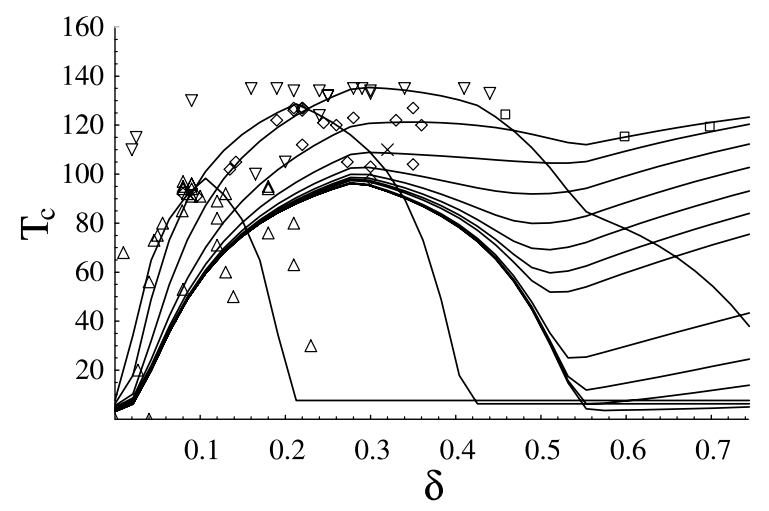

Fig. 9. Modified MNY model: $T_{a}=200, T_{b}=10, \eta=10.2$, $g=0.043, x=0.25$, with $n=1, \ldots, 10,15,20,25,35$. 


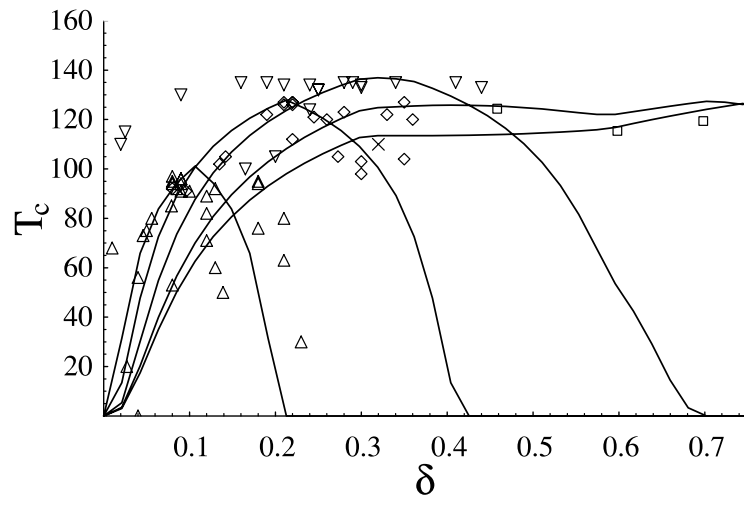

Fig. 10. Modified MNY model: $T_{a}=192, T_{b}=0, \quad \eta=8$, $g=0.043, x=0.3$, with $n=1, \ldots, 5$.

the presence of second maximum for relatively big values $\delta$ and for $n>3$. It is possible to conjecture that the first maximum is connected with the achievement of the optimal value of $p_{0}$ at the outer layers and the second one with the achievement of the optimal value of $p_{0}$ at the inner layers.

The same approximation can be applied to the BL model. This means that the parameters of layers for this model have been taken the same as for the $a$-layers of the MNY model. We summarized these data and the literature data on Fig. 11.

To summarize, in the present section the interplay of the extra oxygen contents $\delta$ and the number of layers $n$ for superconductivity have been investigated. We have considered the model approach for determining the critical temperature depending

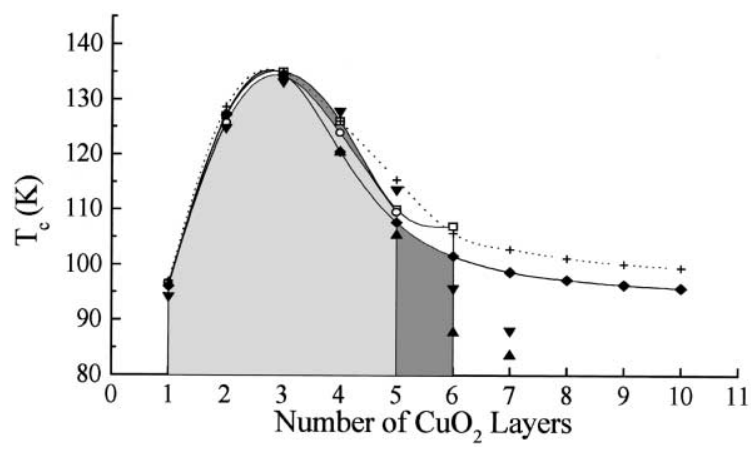

Fig. 11. $T_{\mathrm{c}}$ vs. number of $\mathrm{CuO}_{2}$-layers: $(\boldsymbol{\nabla}) \max ,(\mathbf{\Delta})$ min values [17]; (○) [28]; ( $\square$ ) [10]; (४) MNY model; (+) BL model. on the number of layers $n$ and extra oxygen contents $\delta$. To do this, the redistribution of charge for inequivalent sublayers within the unit cell ("inner" and "outer" layers) was taken into account by minimization of the band and electrostatic energy. The observable nonmonotonic bell-shaped dependence of $T_{\mathrm{c}}(n)$ and $T_{\mathrm{c}}(\delta)$ has been calculated with reasonable agreement with experiment. Dependence of $T_{\mathrm{c}}(\delta)$ is nonmonotonic and shows clearly the maximum which is related with the optimal charge carrier density in the outer layers.

\subsection{Critical temperature and the MNY model}

We consider here, following the line of reasoning of [60], the charge redistribution and dependence $T_{\mathrm{c}}(\delta)$. As has been mentioned above, this model approach may rationalize, at least approximately, the situation in layered mercurocuprate oxides. First, let us summarize the main assumptions of the MNY $m-n$-layered model.

1 . The $b$-type layers are the main charge reservoirs. The outer layers, which are the neighbors of the $a$-layers, transfers more charge than the inner $b$-type layers.

2. $T_{\mathrm{c}}$ of the $b$-type layers is much less than $T_{\mathrm{c}}$ of the $a$-layers. Hence, the two outer layers will give the $x$ carriers (per unit square), while the rest of inner layers will give $c x$ where $c<1$. Finally, all the carriers will be redistributed uniformly among the $a$ - and $b$-layers. The resulting carrier density $\delta(n, m)$ per a single layer will have the form

$\delta(n, m)=\frac{2 x+c x(m-2)}{n+m}$

3. In the model [60], ignoring at the first stage the subtleties of the delicate problem of exact charge redistribution, the following workable anzatz for the critical temperature $T_{a}$ of an $a$-layer dependence on the density of carriers has been adopted

$T_{a}(\delta)=T_{a 0}\left\{1-\left[\frac{\delta-h_{2}}{h_{1}}\right]^{2}\right\}^{1 / 2}$

where $h_{1}$ and $h_{2}$ are the fitting coefficients.

This is a crude approximation and is motivated by the fact that the density-of-states (DOS) varies 
as a function of density of carriers. In the BCS theory of superconductivity, $T_{\mathrm{c}}$ is determined by the pairing interaction strength $g=D\left(\varepsilon_{\mathrm{F}}\right) V$ and $V$ is the pairing potential (in the BCS theory $V$ arises from the electron-phonon interaction). We therefore can write down the variation of $T_{\mathrm{c}}$ with concentration $\delta$ according to

$\frac{\mathrm{d} T_{\mathrm{c}}}{\mathrm{d} \delta}=\frac{\partial T_{\mathrm{c}}}{\partial D\left(\varepsilon_{\mathrm{F}}\right)} \frac{\mathrm{d} D\left(\varepsilon_{\mathrm{F}}\right)}{\mathrm{d} \delta}+\frac{\partial T_{\mathrm{c}}}{\partial V} \frac{\mathrm{d} V}{\mathrm{~d} \delta}$

Only when

$\left|\frac{1}{D\left(\varepsilon_{\mathrm{F}}\right)} \frac{\mathrm{d} D\left(\varepsilon_{\mathrm{F}}\right)}{\mathrm{d} \delta}\right| \gg\left|\frac{1}{V} \frac{\mathrm{d} V}{\mathrm{~d} \delta}\right|$

the change of $T_{\mathrm{c}}$ is mainly related to the change of DOS. In the low carrier density semiconductors $D\left(\varepsilon_{\mathrm{F}}\right) \sim m^{*} p^{1 / 3}$. With these caveats, we may observe that under these approximate conditions, the anzatz (29) is associated with the fact that $T_{\mathrm{c}}$ is not equal to zero in a certain region of changing of $\delta$. In addition, the parameters $\alpha_{a}^{\prime}$ have been considered as constants.

4. Further, the $b$-layers have been supposed to be the weak BCS superconductors. This is possible, in principle, to suppose for the mercurocuprates only, but not for the $\mathrm{Bi}$ - and Tl-based cuprates. The arguments for this were presented in Section 3.1. The energy spectrum of the charge carriers in the infinite stack of the $b$-layers has been taken in [60] in the form

$\varepsilon_{k}=\frac{\hbar^{2}}{2 m}\left(k_{x}^{2}+k_{y}^{2}\right)-2 t_{\perp} \cos k_{z} c_{z}$

where $k=\left(k_{x}, k_{y}, k_{z}\right)$ is the quasi-momentum, $t_{\perp}$ is the nearest neighbor interlayer transfer integral and $c_{z}$ is the interlayer distance. The DOS $D(\varepsilon)$ of this spectrum increases monotonically with the energy $\varepsilon$ for $-2 t_{\perp}<\varepsilon<2 t_{\perp}$ and is nearly constant for $\varepsilon \geqslant 2 t_{\perp}$. For convenience, the origin of energy on $2 t_{\perp}$ has been shifted. In addition, the DOS $D(\varepsilon)$ has been approximated by the linear function for $0<\varepsilon<4 t_{\perp}$.

This form of DOS $D(\varepsilon)$ determines that DOS $D\left(\varepsilon_{\mathrm{F}}\right)$ will increase with $\delta=\delta(n, m)$ when $\delta<m t_{\perp} /$ $\pi \hbar^{2}=\delta_{0}$. For $\delta \geqslant \delta_{0}$ the $\operatorname{DOS} D\left(\varepsilon_{\mathrm{F}}\right)$ will be constant. To relate the critical temperature $T_{b}$ with the carrier concentration $\delta$, the following approximate form can been adopted for rough estimations:
$T_{b}= \begin{cases}T_{b 0}\left(\frac{\delta}{\delta_{0}}\right), & 0<\delta<\delta_{0} \\ T_{b 0}, & \delta \geqslant \delta_{0}\end{cases}$

Taking into account the obvious equality

$\alpha_{b}^{\prime}=\frac{A T_{b}}{\varepsilon_{\mathrm{F}}}$

where $A$ is a constant and the model form of DOS $D(\varepsilon)$, we find $\varepsilon_{\mathrm{F}}$ as a function of $\delta$. Then we substitute $T_{b}=T_{b}(\delta)$ and $\varepsilon_{\mathrm{F}}=\varepsilon_{\mathrm{F}}(\delta)$ into relation (34) to find

$\alpha_{b}^{\prime}(\delta)= \begin{cases}\alpha_{b 0}^{\prime} T_{b 0}\left(\frac{\delta}{\delta_{0}}\right)^{1 / 2}, & 0<\delta<\delta_{0} \\ 2 \alpha_{b 0}^{\prime} T_{b 0} \frac{\delta_{0}}{\delta+\delta_{0}}, & \delta \geqslant \delta_{0}\end{cases}$

where $\alpha_{b 0}^{\prime}=A / 4 t_{\perp}$. We note that the details of the dependence of $\alpha_{b}^{\prime}$ on $\delta$ do not influence substantially on the qualitative behaviour of the critical temperature within this fitting procedure.

5. $\eta$ was taken as a constant.

With these simplifications it is now possible to calculate the critical temperature. There are the following free model parameters:

(i) $n$ and $m$ which determine the structure;

(ii) $x$ and $c$ which determine $\delta$;

(iii) $h_{1}, h_{2}$ and $T_{a 0}$ which determine $T_{a}$;

(iv) $\delta_{0}$ and $T_{b 0}$ which determine $T_{b}$;

(v) $\alpha_{a 0}^{\prime}, \alpha_{b 0}^{\prime}$ and $\eta$.

The essential parameters are: $n, m, T_{a 0}, T_{b 0}, \delta_{0} / x$, $\alpha_{a 0}^{\prime} / \eta, \alpha_{b 0}^{\prime} / \eta, h_{1} / x, h_{2} / x$. Figs. 12 and 13 show the dependence of $T_{\mathrm{c}}(n)$ with changing various

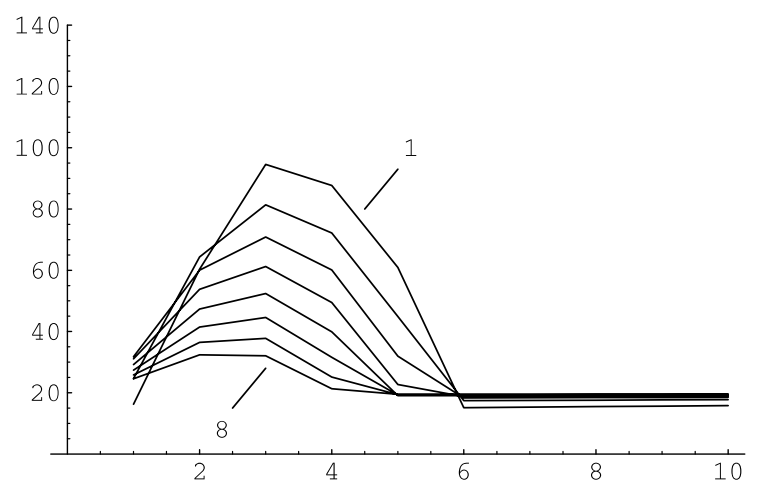

Fig. 12. $T_{\mathrm{c}}(n)$ dependence variation with $m=1,2,3,4,5,6,7,8$. 


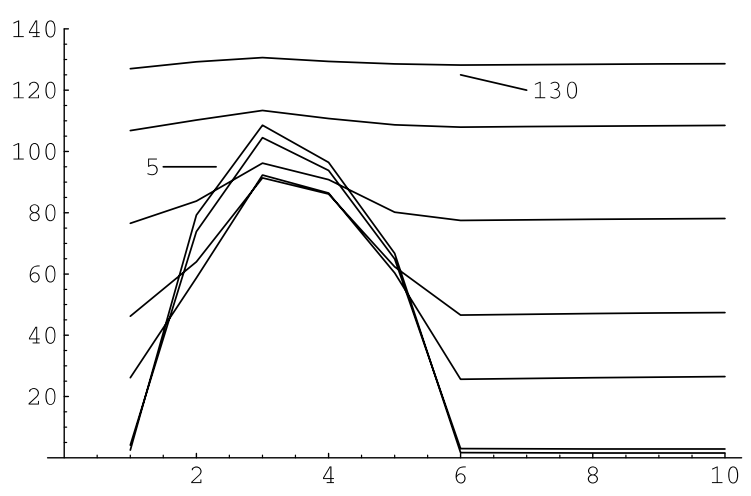

Fig. 13. $T_{\mathrm{c}}(n)$ dependence variation with $\delta_{0}=0.001,0.01,0.07$, $0.1,0.2,0.5$.

parameters. The constant parameters are the following:

- $x=1 ; c=0.2 ; m=1$;

- $h_{1}=0.215 ; h_{2}=0.475 ; T_{a 0}=140$;

- $\delta_{0}=0.1 ; T_{b 0}=20$;

- $\alpha_{a 0}^{\prime}=1.0 ; \alpha_{b 0}^{\prime}=1.0 ; \eta=100$.

After this, we have calculated $T_{\mathrm{c}}(n)$ with $m$ fixed for $m=1,2,3,4$. The results of numerical calculations for $T_{\mathrm{c}}(n)$ and $m=2$ (Fig. 14) are shown as crosses. Thus, incorporating the charge interlayer redistribution, it is possible to restore the observable bell-shaped dependence even in the simplest version of the model and fit the experimental results well.

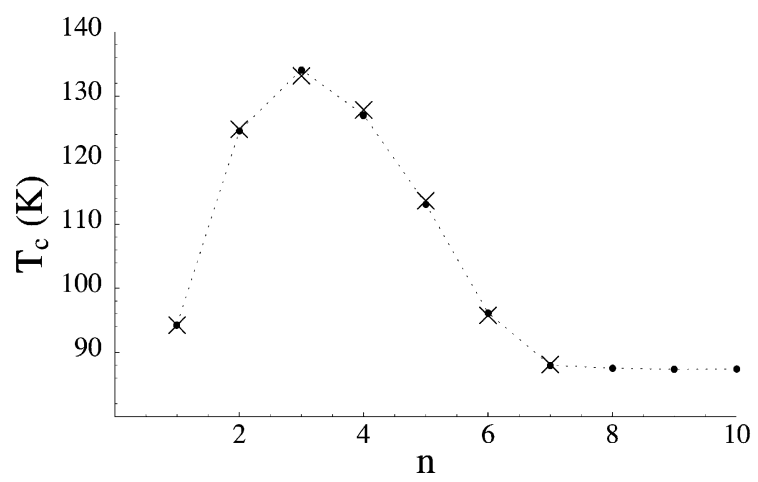

Fig. 14. Dependence of superconducting critical temperature $T_{\mathrm{c}}(n)$ with $m=2$ fixed. Parameters: $T_{a}^{0}=189.5 ; T_{b}^{0}=88.2$; $h_{1}=0.374 ; h_{2}=0.557$.

\section{Conclusions}

In conclusion, in this paper the relations between the crystal structure and superconducting properties of the layered systems (including the homologous series of mercurocuprates) were studied and the factors influencing their superconducting behaviour were analyzed. We discussed the main structural features of layered copper oxides and mercury-based cuprates and gave the physical interpretation and plausible arguments on the role and significance of structural anisotropy and interlayer effects in these substances. To make practical steps, we have used the workable model which rationalizes reasonably the currently available experimental data for the mercurocuprate family. It was shown that within the LD-weak-link type model the observable behaviour could not be explained without an additional invoking the interlayer charge redistribution. Within the framework of our combined model the experimentally observed nonmonotonic bell-shaped dependence $T_{\mathrm{c}}(n)$ was traced back to the experimental one with reasonable accuracy. Therefore, in spite of rather a crude nature of our model approach, the results presented here show that our treatment is quantitatively applicable to the layered systems and, with some reservation, to the mercurocuprate family. On the other side, our analysis favors an approach which is describable in terms of the generalized LD model with suitable charge redistribution corrections. This conclusion should be further substantiated by considering a more general GL model for layered superconductors as well as a more sophisticated description of the charge redistribution. The GL and LD theories are much simpler than the microscopic theory and have a limited domain of validity. However, they give valuable insight into the general physical behaviour and, moreover, they give important parametrization of the systems. In the strategy for searching the factors which increase $T_{\mathrm{c}}$, the number of layers is not the main element, since the single-layer structures, which have been synthesized before mercurocuprates, have $T_{\mathrm{c}}$ in a very wide interval up to about $100 \mathrm{~K}$. Certainly, the number of layers correlates with an increase in $T_{\mathrm{c}}$ in an indirect way. Nevertheless, there is no known microscopic explanation for the 
occurrence of the highest $T_{\mathrm{c}}(n=3)$ observed in the $\mathrm{Hg}-1223$. Further investigations are currently under way to determine the true nature of this phenomenon.

\section{Acknowledgements}

We indebted to E.S. Itskevich, E.V. Antipov and A.M. Balagurov for helpful discussions. A.L.K. thanks A.A. Cheglokov for help in numerical work. I.G.K. was partially supported by the INTAS grant N 991136 and the Russian State program on HTS.

\section{References}

[1] T. Koyama, M. Tachiki, Physica C 193 (1992) 163.

[2] T. Koyama, M. Tachiki, Physica C 210 (1993) 509.

[3] J.R. Clem, Physica A 200 (1993) 118.

[4] K. Kuboya, K. Takanaka, J. Phys. Soc. Jpn. 66 (1997) 3219.

[5] Y. Takano, S. Takayanagi, S. Ogawa, T. Yamadaya, N. Mori, Solid State Commun. 103 (1997) 215.

[6] R.J. Cava, K. Yamaura, S. Loureiro, Q. Huang, R.W. Erwin, J.W. Lynn, D. Young, Physica C 341-348 (2000) 351 .

[7] N.C. Mishra, X. Oudet, Physica C 212 (1993) 465.

[8] S.N. Putilin, E.V. Antipov, O. Chmaissem, M. Marezio, Nature 362 (1993) 226.

[9] E.V. Antipov, S.N. Putilin, E.M. Kopnin, J.J. Capponi, C. Chaillout, M. Marezio, Physica C 235-240 (1994) 21.

[10] S.M. Loureiro, J.J. Capponi, E.V. Antipov, M. Marezio, in: A. Narlikar (Ed.), Studies of High Temperature Superconductors, vol. 25, Nova Science Publ, New York, 1997, p. 229.

[11] H. Yamauchi, M. Karppinen, S. Tanaka, Physica C 263 (1996) 146.

[12] P.P. Edwards, G.B. Peacock, J.P. Hodges, A. Asab, I. Gameson, in: E. Kaldis et al. (Eds.), High- $T_{\mathrm{c}}$ Superconductivity 1996: Ten Years after the Discovery, Kluwer Academic Publ, Dordrecht, 1997, p. 135.

[13] H. Yamauchi, M. Karppinen, Superlatt. Microstruct. A 21 (1997) 127.

[14] H. Yamauchi, M. Karppinen, Mater. Sci. Engng. B 54 (1998) 92.

[15] M. Karppinen, H. Yamauchi, J. Supercond. 11 (1998) 39, 43.

[16] M. Karppinen, H. Yamauchi, Philos. Mag. B 79 (1999) 343.

[17] B.A. Scott, E.Y. Suard, C.C. Tsuei, D.B. Mitzi, T.R. McGuire, B.-H. Chen, D. Walker, Physica C 230 (1994) 239.
[18] R.W. Weast, D.R. Lide (Eds.), Handbook of Chemistry and Physics, CRC Press, Boca Raton, FL, 1998.

[19] J. Karpinski, H. Schwer, E. Kopnin, R. Molinski, G. Meier, K. Conder, J. Hofer, D. Zech, J. Supercond. 11 (1998) 119.

[20] A.M. Hermann, J.V. Yakhmi (Eds.), Thallium-Based High Temperature Superconductors, M. Dekker, Inc., New York, 1994.

[21] H. Maeda, K. Togano (Eds.), Bismuth-Based High Temperature Superconductors, M. Dekker, Inc., New York, 1996.

[22] E.S. Itskevich, T.I. Dyuzheva, I.G. Kuzemskaya, K.A. Lokshin, JETP Lett. 62 (1995) 512.

[23] I.G. Kuzemskaya, E.S. Itskevich, L.F. Kulikova, K.A. Lokshin, J. Low Temp. Phys. 105 (1996) 1565.

[24] E.S. Itskevich, T.I. Dyuzheva, I.G. Kuzemskaya, K.A. Lokshin, Inorg. Mater. 33 (1997) 404.

[25] K.A. Lokshin, I.G. Kuzemskaya, L.F. Kulikova, E.V. Antipov, E.S. Itskevich, Physica C 279 (1997) 11.

[26] M.-S. Kim, W.-S. Kim, S.-I. Lee, S.-C. Yu, E.S. Itskevich, I.G. Kuzemskaya, Physica C 282-287 (1997) 1987.

[27] I.G. Kuzemskaya, K.A. Lokshin, L.F. Kulikova, E.V. Antipov, E.S. Itskevich, J. Supercond. 11 (1998) 117.

[28] K.A. Lokshin, D.A. Pavlov, M.L. Kovba, E.V. Antipov, I.G. Kuzemskaya, L.F. Kulikova, V.V. Davydov, I.V. Morozov, E.S. Itskevich, Physica C 300 (1998) 71.

[29] M.-S. Kim, S.-I. Lee, S.-C. Yu, I.G. Kuzemskaya, E.S. Itskevich, K.A. Lokshin, Phys. Rev. B 57 (1998) 6121.

[30] K. Fujinami, T. Ito, H. Suematsu, K. Matsuura, M. Karppinen, H. Yamauchi, Phys. Rev. B 56 (1997) 14790.

[31] A. Fukuoka, A. Tokiwa-Yamamoto, M. Itoh, R. Usami, S. Adashi, K. Tanabe, Phys. Rev. B 55 (1997) 6612.

[32] E.S. Itskevich, V.P. Kraidenov, I.G. Kuzemskaya, JETP 91 (2000) 562.

[33] J.L. Wagner, P.G. Radaelli, D.G. Hinks, J.D. Jorgensen, J.F. Mitchel, B. Dabrowski, G.S. Knapp, M.A. Beno, Physica C 210 (1993) 447.

[34] A. Bertinotti, D. Colson, J. Hammann, J.F. Marucco, D. Luzet, A. Pinatel, V. Viallet, Physica C 250 (1995) 213.

[35] L.W. Finger, R.M. Hazen, R.T. Downs, R.L. Meng, C.W. Chu, Physica C 226 (1994) 216.

[36] D. Colson, A. Bertinotti, J. Hammann, J.F. Marucco, A. Pinatel, Physica C 233 (1994) 231.

[37] A. Tokiwa-Yamamoto, K. Isawa, M. Itoh, S. Adashi, H. Yamauchi, Physica C 216 (1993) 250.

[38] E.V. Antipov, A.M. Abakumov, V.A. Alyoshin, K.A. Lokshin, D.A. Pavlov, D.A. Mikhailova, S.N. Putilin, M.G. Rozova, A.M. Balagurov, I.G. Kuzemskaya, E.S. Itskevich, in: K. Van Tendeloo et al. (Eds.), HighTemperature Superconductors and Novel Inorganic Materials, Kluwer Publ, Dordrecht, 1999, p. 157.

[39] J. Karpinski, H. Schwer, K. Conder, G.I. Meijer, E. Kopnin, R. Molinski, Solid State Ionics 101-103 (1997) 985.

[40] J. Karpinski, H. Schwer, R. Molinski, G.I. Meijer, K. Conder, E. Kopnin, J. Lohle, C. Rossel, D. Zech, J. Hofer, A. Wisniewski, R. Puzniak, in: A. Narlikar (Ed.), Studies 
of High Temperature Superconductors, vol. 24II, Nova Science Publ, NY, 1998, p. 165.

[41] Q. Huang, J.W. Lynn, Q. Xiong, C.W. Chu, Phys. Rev. B 52 (1995) 462.

[42] J.L. Wagner, B.A. Hanter, D.G. Hinks, J.D. Jorgensen, Phys. Rev. B 51 (1995) 15407.

[43] V.L. Aksenov, A.M. Balagurov, V.V. Sikolenko, V.G. Simkin, V.A. Alyoshin, E.V. Antipov, A.A. Gippius, D.A. Mikhailova, S.N. Putilin, F. Bouree, Phys. Rev. B 55 (1997) 3966.

[44] A.M. Abakumov, V.L. Aksenov, V.A. Alyoshin, E.V. Antipov, A.M. Balagurov, D.A. Mikhailova, S.N. Putilin, M.G. Rozova, Phys. Rev. Lett. 80 (1998) 385.

[45] A.M. Balagurov, D.V. Sheptyakov, V.L. Aksenov, E.V. Antipov, S.N. Putilin, P.G. Radaelli, M. Marezio, Phys. Rev. B 59 (1999) 7209.

[46] Zs. Gulacsi, M. Gulacsi, I. Pop, Phys. Rev. B 37 (1988) 2247.

[47] J. Labbe, J. Bok, Europhys. Lett. 3 (1987) 1225.

[48] J. Bok, Solid State Commun. 67 (1988) 251.

[49] D. Baeriswyl, T. Schneider, Int. J. Mod. Phys. B 1 (1988) 523.

[50] K. Byczuk, J. Spalek, Phys. Rev. B 53 (1996) R518.
[51] E.S. Itskevich, E.V. Zhasinas, Physica C 295 (1998) 193.

[52] D. Tristan Jover, R.J. Wijngaarden, H. Wilhelm, R. Griessen, S.M. Loureiro, J.J. Capponi, A. Schilling, H.R. Ott, Phys. Rev. B 54 (1996) 4265.

[53] S. Theodorakis, Z. Tesanovic, Phys. Lett. A 132 (1988) 372 .

[54] S. Theodorakis, Phys. Lett. A 132 (1988) 287.

[55] A.K. Setty, Physica C 215 (1993) 113.

[56] T. Koyama, N. Takezawa, Y. Naruse, M. Tachiki, Physica C 194 (1992) 20.

[57] J. Birman, J.P. Lu, Phys. Rev. B 39 (1989) 2238.

[58] W.E. Lawrence, S. Doniach, in: E. Kanda (Ed.), Proc. of the 12th Int. Conf. on Low Temp. Phys. 1970, Tokyo, 1971 , p. 361.

[59] J.B. Ketterson, S.N. Song, Superconductivity, Cambridge University Press, Cambridge, 1999.

[60] H. Mori, T. Nagao, F. Yonezawa, J. Phys. Soc. Jpn. 58 (1989) 2501.

[61] M. Di Stasio, K.A. Muller, L. Pietronero, Phys. Rev. Lett. 64 (1990) 2827.

[62] E.M. Haines, J.L. Tallon, Phys. Rev. B 45 (1992) 3172.

[63] R.P. Gupta, M. Gupta, Physica C 223 (1994) 213.

[64] M.S. Islam, L.J. Winch, Phys. Rev. B 52 (1995) 462. 